\title{
Systemic low-grade inflammation in post- traumatic stress disorder: a systematic review
}

This article was published in the following Dove Press journal: Journal of Inflammation Research

\section{Kathryn Speer ${ }^{1}$ \\ Dominic Upton ${ }^{2}$ \\ Stuart Semple ${ }^{1,3}$ \\ Andrew McKune ${ }^{1-4}$}

'Discipline of Sport and Exercise Science, Faculty of Health, University of Canberra, Canberra, ACT, Australia; ${ }^{2}$ Faculty of Health, University of Canberra, Canberra, ACT, Australia;

${ }^{3}$ Research Institute for Sport and Exercise, University of Canberra, Canberra, ACT, Australia; ${ }^{4}$ Discipline of Biokinetics, Exercise and Leisure Sciences, School of Health Sciences, University of KwaZulu Natal, Durban, South Africa
Correspondence: Kathryn Speer University of Canberra Research Institute for Sport and Exercise (UCRISE), Building 29, University of Canberra, Bruce, ACT 260I, Australia Tel +6I 403844963 Email kate.e.speer@gmail.com

\begin{abstract}
Studies examining post-traumatic stress disorder (PTSD) have either emphasized a relationship between PTSD and a systemically pro-inflammatory state or identified a link between PTSD and chronic disease. The aim of this study was to evaluate the evidence for a relationship between individuals with PTSD and systemic low-grade inflammation that has been proposed to underlie chronic disease development in this population. The authors conducted a systematic review of the literature (January 2006 to April 2017) in accordance with the PRISMA statement in the following four databases: PubMed, MEDLINE, PsycINFO, and SPORTDiscus with Full Text. The search strategy was limited to articles published in peer-reviewed journals and to human studies. Nine studies measuring systemic inflammation and discussing its role in chronic disease development were selected for inclusion in this review. The association between markers of systemic inflammation and PTSD was evaluated by the measurement of a variety of systemic inflammatory markers including acute-phase proteins, complement proteins, pro- and anti-inflammatory cytokines, natural killer cells, and white blood cells. In general, systemic inflammatory biomarkers were elevated across the studies in the PTSD groups. There is evidence that PTSD is underpinned by the presence of a systemic low-grade inflammatory state. This inflammation may be the mechanism associated with increased risk for chronic disease in the PTSD population. From this, future research should focus on interventions that help to reduce inflammation, such as exercise.
\end{abstract}

Keywords: cytokines, C-reactive protein, CRP, chronic disease, pro-inflammation, immune system

\section{Introduction}

Post-traumatic stress disorder (PTSD) is a debilitating psychiatric condition that manifests after experiencing trauma. Defined as an Axis I disorder in the Diagnostic and Statistical Manual of Mental Disorders, fifth edition, PTSD is characterized by a number of symptoms such as re-experiencing the traumatic event, avoidance of reminders of the traumatic event, negative changes in mood and cognitions, and hyperarousal. ${ }^{1,2}$ These symptoms must persist and cause marked distress and/or functional impairment for at least one month. ${ }^{1}$ The lifetime prevalence rate for PTSD is estimated to be $8 \%$, making it the fourth most commonly diagnosed psychiatric disorder, with $12 \%$ and $18 \%$ of US soldiers suffering from PTSD upon their return from Afghanistan and Iraq, respectively. ${ }^{3,4}$ Similarly, among Australian war veterans, PTSD is the most commonly diagnosed mental health disorder, with a prevalence of $8.1 \%$ in Australian male soldiers. ${ }^{5}$ 
PTSD places a significant burden on the individual, his or her family, and society at large, as not only is it associated with multiple negative social consequences such as marital difficulties and unemployment but also it seems to be correlated with an increased risk of developing chronic somatic diseases such as cardiovascular disease (CVD), metabolic syndrome, pulmonary disease, autoimmune disorders, and other psychiatric disorders that are often associated with PTSD. ${ }^{2,6-8}$ Notably, low-grade systemic inflammation is seen as a key mechanism underlying chronic disease and may be present too in PTSD, leading to the development of chronic diseases in this population. ${ }^{2,9}$

The underlying psychobiological mechanisms linking PTSD to an increased chronic disease risk have been proposed to be altered autonomic nervous system (ANS) activity and an impaired reaction of the hypothalamic-pituitary-adrenal (HPA) axis to perceived stress, resulting in a chronic low-grade pro-inflammatory state. ${ }^{10}$ In the context of a chronically hyperaroused state (as seen in individuals suffering from PTSD), the ANS has been shown to display an inappropriate response characterized by a delay in parasympathetic nervous system (PNS) reactivation and sympathetic nervous system (SNS) domination continuing long after the stressful event has dissipated. ${ }^{11}$ The consequence of prolonged autonomic reactivity in PTSD patients may center around the bi-directional relationship between the ANS and HPA axis, as represented by vagal activity. ${ }^{2}$ Considering that a function of the vagus nerve is to regulate the immune system, an attenuated vagal response would fail to re-establish PNS dominance at rest. This, in turn, may compromise the normal immune profile, exacerbating the risk for chronic disease. ${ }^{2}$ In addition, the dysregulated HPA axis may contribute to a chronic low-grade inflammatory state due to alterations within the glucocorticoid (GC) receptor itself and reduced GC signaling. Changes to GC responsiveness may also contribute to allostatic load, resulting in chronic disease development. ${ }^{12}$

Dantzer and colleagues ${ }^{13}$ proposed that systemic lowgrade inflammation is typically defined by a two- to threefold increase in plasma concentrations of pro-inflammatory cytokines, such as interleukin (IL)-1, tumor necrosis factor- $\alpha$ (TNF- $\alpha$ ), and IL-6. ${ }^{14,15}$ However, the origin of this low-grade inflammation remains unclear. Neigh and $\mathrm{Ali}^{16}$ proposed that PTSD leads to a pro-inflammatory state as a consequence of the dysregulations in the aforementioned ANS and HPA axis biological pathways, thereby increasing physical disease risk. Conversely, Walker et $\mathrm{al}^{17}$ suggested an alternative hypothesis for developing PTSD, emphasizing that chronic occupational exposures such as physical exhaustion, smoke, heat exposure, and sleep restriction (mainly experienced by military veterans and first responders who regularly encounter traumatic events) may have a "priming" inflammatory effect for the later development of PTSD, ultimately leading to chronic somatic illness.

However, this proposed state of low-grade inflammation characteristic of both PTSD and chronic disease is not always indicated. While various studies have demonstrated elevations in inflammatory markers in PTSD sufferers, others have presented opposing findings. ${ }^{6,18-25}$ In light of these discrepancies, the aim of this systematic review was to assess the research that examines the association between PTSD and low-grade systemic inflammation.

\section{Material and methods}

This systematic review was conducted in accordance with the established guidelines from the Preferred Reporting Items for Systematic Reviews and Meta-Analyses (PRISMA) Statement. ${ }^{26,27}$

\section{Search strategy}

An electronic literature search of peer-reviewed journal articles was conducted using four databases: PubMed, MEDLINE, PsycINFO, and SPORTDiscus. Medical Subject Headings (MeSH) were considered in the development of the search terms. The search was limited to the English language and to human studies. Studies from before 2006 were excluded. The lead author screened titles, abstracts, and methods for relevance based on the selection criteria, and all duplicates were removed. Those articles deemed relevant were selected for further consideration (Figure 1). The search was further limited to peer-reviewed original research articles with full text available. Reviews, metaanalyses, and other types of paper (e.g. author manuscripts, letters to the editor) were excluded. A hand search was undertaken of the reference lists of relevant articles, and those deemed eligible were included with the intent to ensure that all articles relevant to the research had been captured by the search strategy. The abstracts of the articles deemed relevant by the lead author were then independently reviewed by a second investigator for relevance. If consensus was not reached, the article was moved on to the next stage for a review of the full text. The full texts of the remaining eligible studies were independently reviewed by the investigators against the inclusion and exclusion 


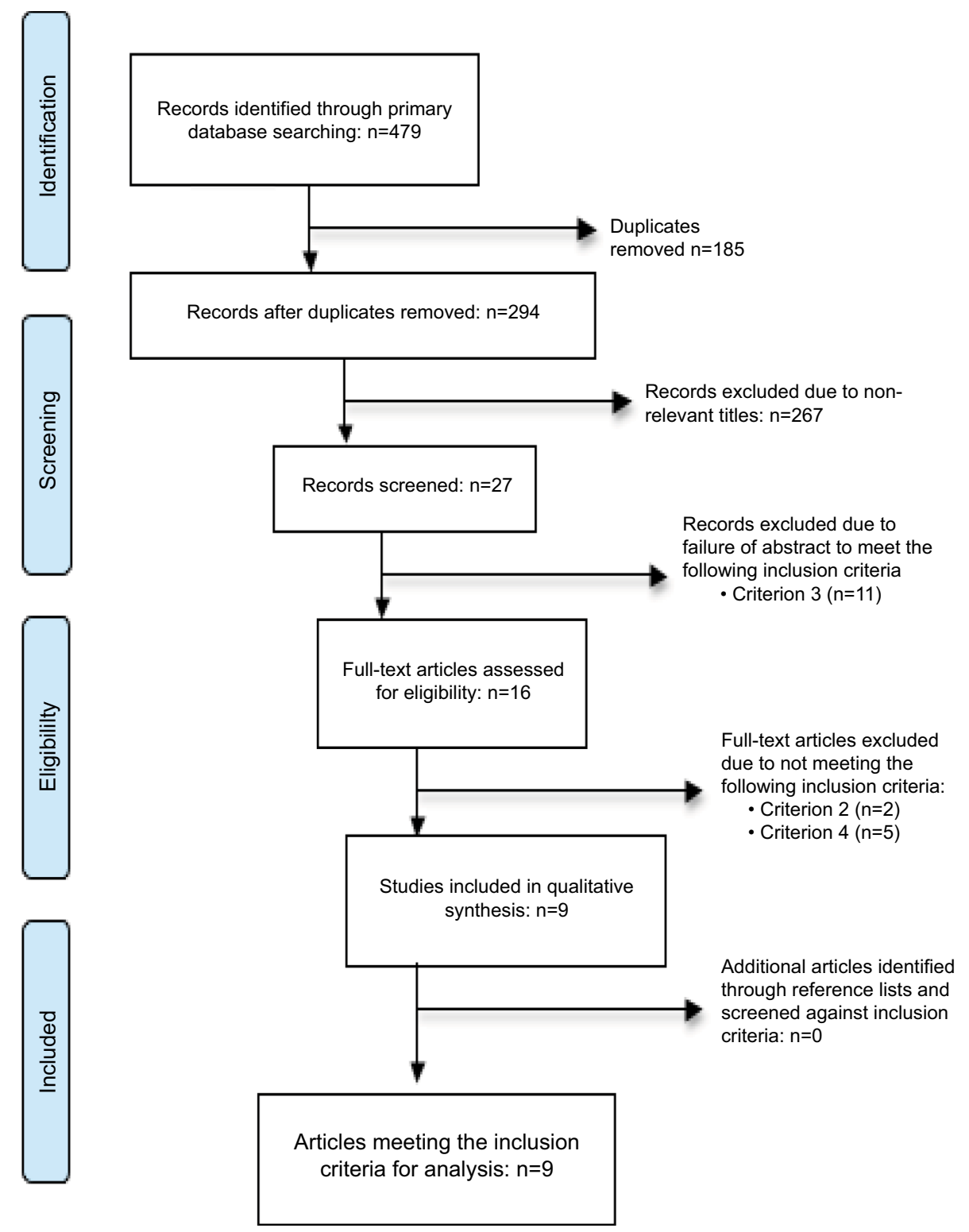

Figure I Schematic representation of the flow of information during the different phases of the systematic review.

criteria. Disagreements were discussed until consensus was reached in all cases.

\section{Search terminology}

The search terms used included: "post-traumatic stress disorder" OR "PTSD," AND “cardiovascular disease" OR “CVD” OR "physical health" OR "metabolic disease" OR "immune system" OR "inflammation" OR "C-reactive protein" OR "CRP” OR "cytokines" OR "leukocytes" OR "white blood cells" OR "WBC” OR "acute phase proteins" OR “complement" OR "innate immunity" OR "hypothalamic pituitary adrenal axis" OR "HPA axis." These were combined using the Boolean operators as stated above.

\section{Selection criteria and data extraction}

Studies were eligible for inclusion if the following applied: (1) they examined trauma leading to PTSD; (2) they examined the relationship between PTSD, inflammation, and chronic disease risk; (3) they had a correlational or longitudinal design (observational, randomized trials); (4) they had a control group; and (5) they measured at least one systemic inflammatory biomarker.

All papers identified from the initial electronic search process were imported into EndNote (version X7; Thomson Reuters), where all duplicates were removed. Upon completion of the title and abstract reviews, a total of 16 articles were identified for full-text review, of which nine were identified 
as meeting the inclusion criteria. The reasons for excluding each article were recorded.

\section{Data analysis}

After study selection, criteria were followed to maintain the evaluation of the studies within narrow standards. The first and essential criterion was that all details regarding inflammatory marker collection should be verified, with description of the instruments, data collection protocol, and methods.

Data extraction of included studies was completed by two independent reviewers and compiled into a table. This included the following: author and year of publication, number of participants, study design, focus of the study, the inflammatory markers tested, and the statistical significance of study findings.

\section{Risk of bias}

Two independent authors used the Physiotherapy Evidence Database (PEDro) scale to assess the quality and risk of bias of each of the studies identified as eligible (Table 1). The PEDro scale has proven constructive validity. ${ }^{28}$ It is an 11-item scale that is used to assess the quality of the methodology in studies..$^{29,30}$ The PEDro scale measures/ assesses the eligibility criteria, randomization, allocation processes, similarity of baseline groups, blinding process, near-complete data sets obtained for the key outcome, between-group statistics reported, and measures of variability in the selected studies. ${ }^{29}$ Criterion 1 is used to help determine the external validity of the study; criteria 2-9 are designed to help identify whether the study has internal validity; and criteria 10 and 11 assist in determining whether there is sufficient statistical information for the results to be interpreted appropriately. ${ }^{29}$ Each of these items was allocated a score in relation to the study. A point was allocated if the study met the specified criterion and a zero was awarded when the item did not. A total score of bias for the study was then calculated by adding all of the items together. The risk of bias for the studies was established using the criteria from the PEDro scale, summating items 2-4 and 7-9. The six criteria that were used in the current review were: (i) adequacy of randomization (criterion 2); (ii) allocation concealment (criterion 3); (iii) between-group baseline comparability (criterion 4); (iv) blinding of outcome assessors (criterion 7); (v) adequate follow-up (more than 85\%) (criterion 8); and (vi) intention-to-treat analysis (criterion 9). A score of 5 or 6 was considered to have a low risk of bias, 3 or 4 a moderate risk, and 2 or less a high risk.

\section{Results}

\section{Study design and characteristics}

In total, 479 records were screened, after the data had been filtered and duplicates removed. From these, nine studies were identified as meeting the inclusion criteria (Figure 1). All of the selected studies were of a cross-sectional design. Data are presented in Table 2 with a description of the studies (including population and design) according to their focus relating inflammation to chronic physical disease in PTSD sufferers, the variables that were tested (inflammatory markers) and the significant statistical findings.

The purpose of all included studies was to investigate the systemic inflammatory status of individuals affected by PTSD.

Table I PEDro scale outcomes

\begin{tabular}{|c|c|c|c|c|c|c|c|c|c|c|c|c|c|}
\hline \multirow[t]{2}{*}{ Reference } & \multicolumn{11}{|c|}{ Criterion } & \multirow[t]{2}{*}{ Total } & \multirow[t]{2}{*}{ Risk of bias } \\
\hline & $\mathrm{I}$ & 2 & 3 & 4 & 5 & 6 & 7 & 8 & 9 & 10 & 11 & & \\
\hline Lindqvist et al $(20 \text { I } 7)^{6}$ & $x$ & 0 & 0 & $x$ & 0 & 0 & 0 & $x$ & $x$ & $x$ & $x$ & $6 / 11$ & $3 / 6$ (moderate) \\
\hline Lindqvist et al $(20 \text { l } 4)^{23}$ & $x$ & 0 & 0 & $x$ & 0 & 0 & 0 & $x$ & $x$ & $x$ & $x$ & $6 / 11$ & $3 / 6$ (moderate) \\
\hline Heath et al $(2013)^{20}$ & $x$ & 0 & 0 & 0 & 0 & 0 & 0 & $x$ & $x$ & $x$ & $x$ & $5 / 11$ & 2/6 (high) \\
\hline Gola et al $(2013)^{25}$ & $x$ & 0 & 0 & $x$ & 0 & 0 & 0 & $x$ & $x$ & $x$ & $x$ & $6 / 11$ & $3 / 6$ (moderate) \\
\hline Spitzer et al $(2010)^{24}$ & $x$ & 0 & 0 & $x$ & 0 & 0 & 0 & $x$ & $x$ & $x$ & $x$ & $6 / 11$ & $3 / 6$ (moderate) \\
\hline Hoge et al (2009) & $x$ & 0 & 0 & $x$ & 0 & 0 & 0 & $x$ & $x$ & $x$ & $x$ & $6 / 11$ & $3 / 6$ (moderate) \\
\hline von Känel et al (2007) ${ }^{18}$ & $x$ & 0 & 0 & $x$ & 0 & 0 & 0 & $x$ & $x$ & $x$ & $x$ & $6 / 11$ & $3 / 6$ (moderate) \\
\hline Hovhannisyan et al $(2010)^{22}$ & $x$ & 0 & 0 & $x$ & 0 & 0 & 0 & $x$ & $x$ & $x$ & $x$ & $6 / 11$ & $3 / 6$ (moderate) \\
\hline Bersani et al $(2016)^{19}$ & $x$ & 0 & 0 & $x$ & 0 & 0 & 0 & $x$ & $x$ & $x$ & $x$ & $6 / 11$ & $3 / 6$ (moderate) \\
\hline
\end{tabular}

Notes: Criteria: I, eligibility criteria were specified; 2, subjects were randomly allocated to groups (in a crossover study, subjects were randomly allocated an order in which treatments were received); 3 , allocation was concealed; 4 , the groups were similar at baseline regarding the most important prognostic indicators; 5 , there was blinding of all subjects; 6, there was blinding of all therapists who administered the therapy; 7, there was blinding of all assessors who measured at least one key outcome; 8 , measures of at least one key outcome were obtained from more than $85 \%$ of the subjects initially allocated to groups; 9 , all subjects for whom outcome measures were available received the treatment or control condition as allocated or, where this was not the case, data for at least one key outcome were analyzed by "intention to treat"; 10 , the results of between-group statistical comparisons are reported for at least one key outcome; II, the study provides both point measures and measures of variability for at least one key outcome. Data from Verhagen et al. ${ }^{29}$

Abbreviation: PEDro, Physiotherapy Evidence Database. 


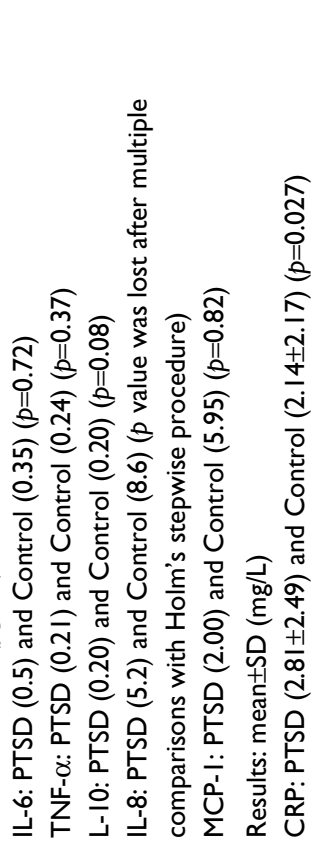

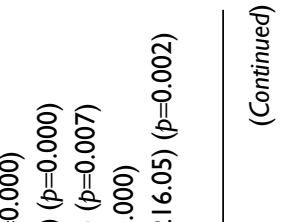

iI

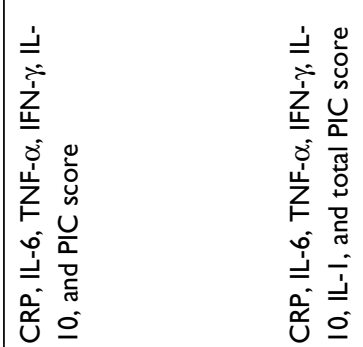

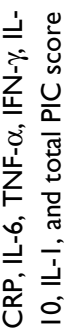
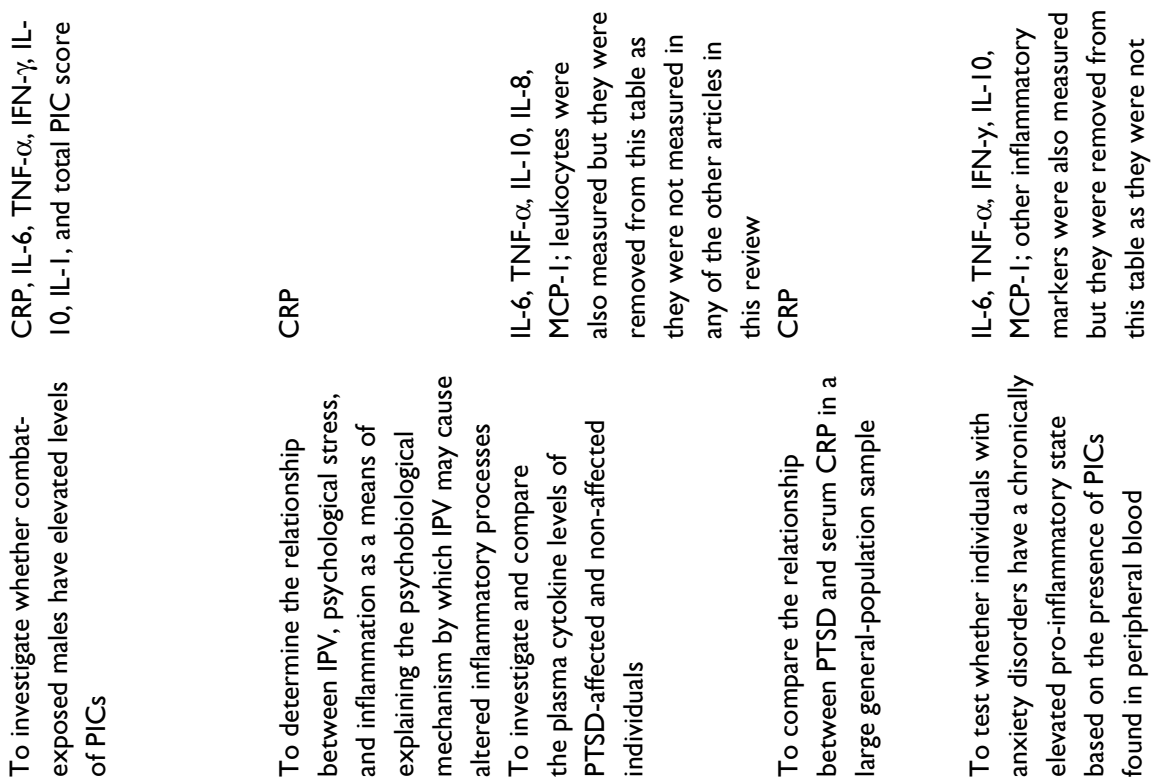

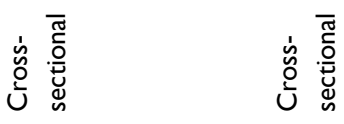

蛋

ธิ

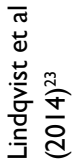

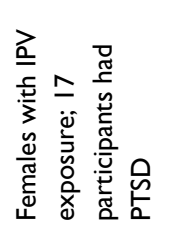

$\stackrel{\circ}{\underline{m}}$

岁高

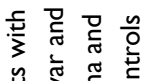

离 气

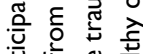

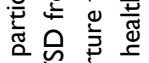

品占产

8

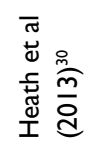

离

峞

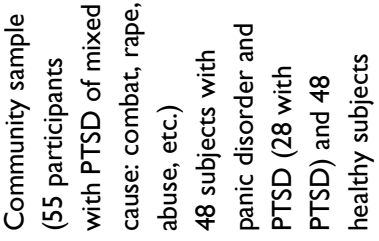

ণ্े

2

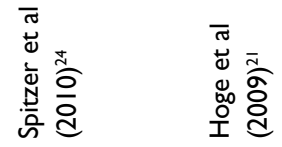

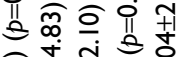

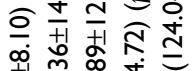

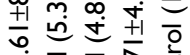

这

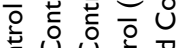

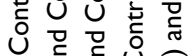

ग

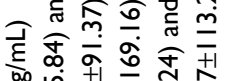

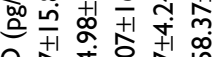

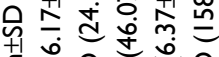

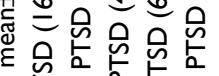

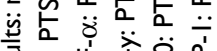

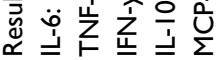

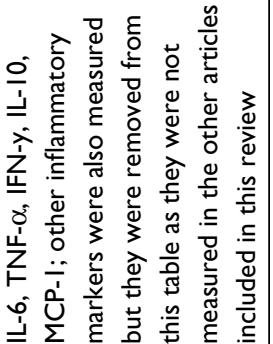

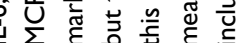




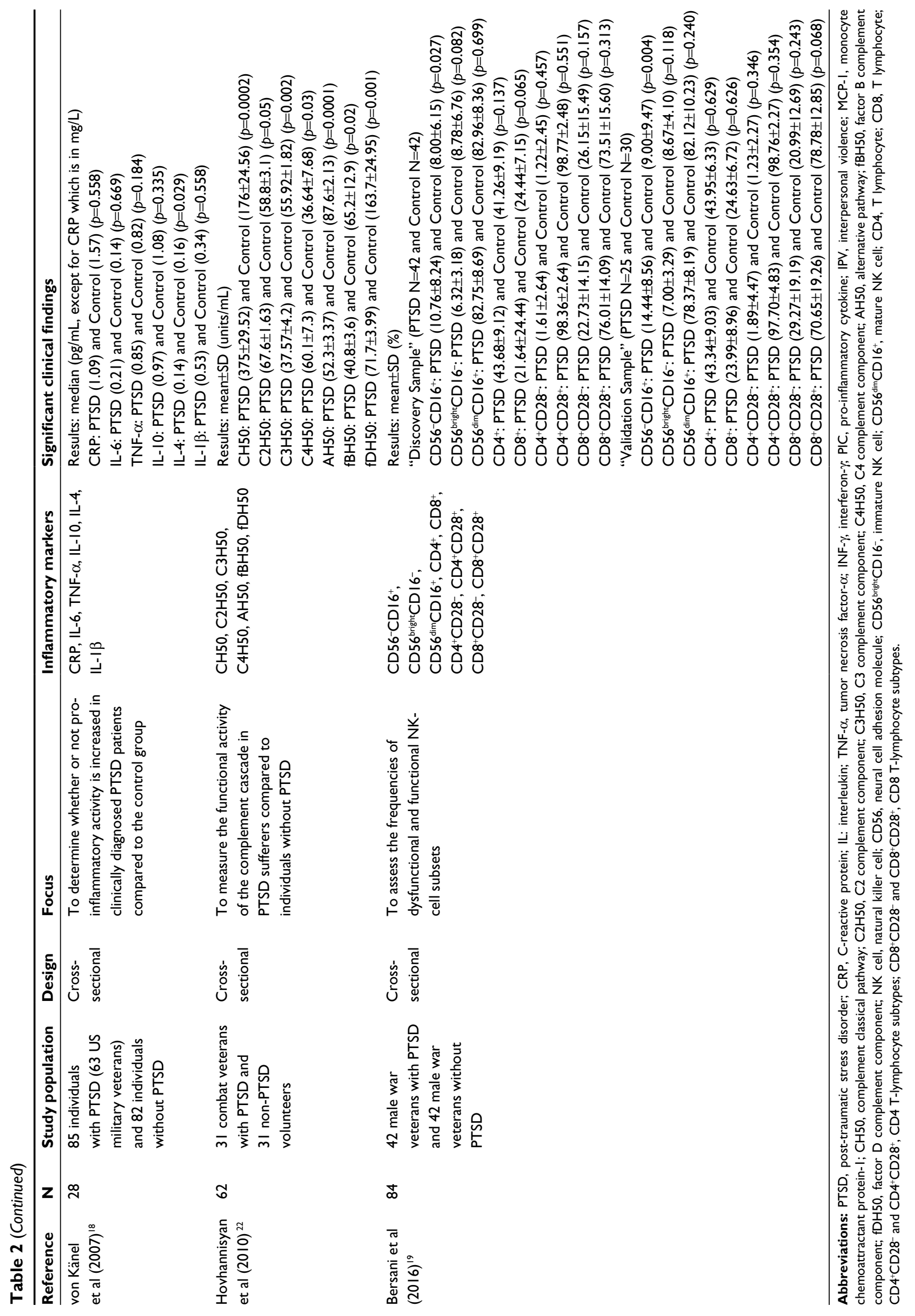




\section{Populations assessed}

The PTSD populations included in the nine studies included in this analysis comprised military veterans and individuals who had experienced traumatic accidents or interpersonal violence such as physical and sexual assault, although one article did not specify the source of trauma. ${ }^{6,18-25}$ Some studies included only male subjects, whereas most consisted of males and females, and only one included just females. ${ }^{6,18-25}$ All studies included in this review evaluated various cytokines to portray the inflammatory state of the PTSD group against that of the control group. Out of these nine studies, all but one demonstrated a statistically significant $(p \leq 0.05)$ difference in at least one inflammatory marker in the PTSD compared to the control group, with the PTSD population displaying an elevated level of pro-inflammatory cytokines, acute-phase proteins, and/or white blood cells. ${ }^{25}$ The research conducted by Gola and co-workers ${ }^{25}$ did not reveal statistically significant intergroup differences in basal concentrations of IL-6, IL-8, IL-10, TNF- $\alpha$, or monocyte chemoattractant protein-1 (MCP-1).

\section{Control for confounding factors}

Through the inclusion and exclusion criteria and statistical analyses, the reviewed studies controlled for the following confounding factors that have known implications on the validity of inflammatory marker results: age, ${ }^{6,18-24}$ gender, ${ }^{6,18-25}$ psychiatric co-morbidities, ${ }^{6,18-21,23,25}$ medication, ${ }^{6,19,21-25}$ asthma/allergies, ${ }^{6,23}$ ethnicity, ${ }^{6,19-21,23,25}$ education, ${ }^{6,19,20,23}$ length of time since trauma, ${ }^{6,18,19,23}$ body mass index (BMI), ${ }^{6,18-21,23,24}$ type of trauma, ${ }^{6,19,23,25}$ alcohol/substance abuse, ${ }^{18,19,24,25}$ smoking status, ${ }^{6,18,19,21,23-25}$ physical activity, ${ }^{18,24}$ employment, ${ }^{20}$ physical disease, ${ }^{6,18,19,22-25}$ marital status, ${ }^{20}$ and early life trauma. ${ }^{6,23}$

\section{Definitions of low-grade inflammation}

All studies included in this review conducted research investigating the association between PTSD and an increased chronic disease risk. Each study aimed to provide physiological evidence for this relationship by measuring biological inflammatory markers of individuals suffering from PTSD versus a control group. However, only three out of nine studies clearly defined low-grade inflammation (C-reactive protein $[\mathrm{CRP}]>3 \mathrm{mg} / \mathrm{L} ; 3$ standard deviations $>$ mean value of IL-6, IL-8, IL-10, TNF- $\alpha$, and MCP-1). ${ }^{6,24,25}$ All of the included studies compared between-group inflammatory cytokine levels, acute-phase proteins, and/ or white blood cells, and tested for statistical significance $(p \leq 0.05)$.

\section{Discussion}

Normal physiological responses to stress are disrupted in individuals suffering from PTSD, as evidenced by an altered response from the ANS (attenuation of vagal tone resulting in a delayed reactivation of the PNS and sympathetic tone domination) and an impaired HPA axis reaction to ongoing stress (inhibition of the HPA axis negative feedback loop involving cortisol). ${ }^{16}$ Dennis and co-workers ${ }^{2}$ emphasized that the synchronicity underlying the suggested interaction between these two systems is vital to maintaining healthy adaptive physiological functioning. Disruptions in these biological pathways (as noted in PTSD-affected people) appear to have downstream effects on other bodily systems such as the immune system, as illustrated by chronic low-grade inflammation, which may ultimately lead to a higher risk of developing a number of physical diseases. ${ }^{16,31}$

While research has identified the possible biological mechanistic impairments that seem to be characteristic of PTSD, disagreements remain regarding the potential catalysts for developing PTSD. Specifically, it has not been established whether an individual is "primed" to develop PTSD through an already existing pro-inflammatory state or if chronic low-grade inflammation manifests after the development of PTSD. ${ }^{17,32}$ Furthermore, there are discrepancies between previous studies' findings of various inflammatory markers compared to a control group. However, while these inconsistencies are apparent, it is inarguable that there is a significant association between PTSD, inflammation, and declining physical health.

In addition to the altered levels of inflammatory markers in PTSD sufferers compared to their non-PTSD counterparts, all reviewed studies identified a clear risk and prevalence of physical co-morbidities of the participants with PTSD. Moreover, these studies interpreted chronic inflammation (measured through acute-phase proteins such as CRP and complement proteins, pro- and anti-inflammatory cytokine levels, innate immune cells, and/or white blood cells) as the link tying PTSD to chronic disease risk, with CVD having the highest prevalence compared to other chronic diseases in this population..$^{33}$ Furthermore, all investigators cited an urgent need for the development of interventions that address inflammation and chronic disease in PTSD, as its physical comorbidities reduce life expectancy by $10-17$ years. ${ }^{34}$

Given the importance of improving the debilitating comorbid chronic physical health conditions of PTSD, this review contributed by investigating the evidence for systemic inflammation in PTSD to substantiate the proposed link between PTSD and chronic disease. Despite the majority of 
the studies demonstrating significant between-group differences in inflammatory markers, these findings were not unanimous. In addition, there was a limited number of studies that met the criteria for this review, all of a cross-sectional design, and they were not without multiple confounders. Out of the nine studies included in this review, one had a relatively small $(n=28)$ sample size and all studies had either a moderate or high risk of bias according to the PEDro scale. ${ }^{18}$ Furthermore, there were clear differences in the populations comprising the control groups between studies. Some studies controlled for trauma exposure in both groups, while the non-PTSD groups in the remaining studies included participants who had no trauma record whatsoever. ${ }^{6,18-25}$ As a consequence, regarding the studies that did not have a trauma-exposed control group, the notion that trauma exposure is the factor affecting inflammation cannot be dismissed. ${ }^{18}$ Similarly, only three studies took into account timing of exposure to the trauma and none of the studies investigated the possible effect of the duration of participants' PTSD status on systemic inflammation. ${ }^{6,22,23}$ Having said that, one of the reviewed studies measured the effect of repeated exposure to trauma on inflammation in PTSD participants and concluded that the number of traumatic exposures was directly proportional to systemic inflammation. ${ }^{25}$

As there is a multitude of variables to account for in psychoneuroimmunological research, this review recognizes that demonstrating psychobiological relationships and the consequential effects if their underlying mechanisms are impaired is relatively new; as such, the investigations included in this review have provided crucial information regarding preliminary psychological and physiological information in regard to systemic low-grade inflammation and chronic disease prevalence in the PTSD-afflicted population. ${ }^{35}$ These studies have established the foundation upon which further research can build as a means to provide individuals suffering from PTSD with novel, long-term, effective treatment plans not only to improve the psychological symptoms of PTSD but also to reduce the physical morbidity and early mortality rates demonstrated in this population. ${ }^{36}$ The universal theme embedded across the reviewed studies was the clear association between PTSD and systemic low-grade inflammation.

\section{PTSD and inflammation}

The major finding regarding the association between inflammation and PTSD was the significant difference in specific pro- and anti-inflammatory cytokines, acute-phase proteins, innate immune cells, and/or white blood cells between the PTSD-affected and unaffected, "healthy" groups. The most commonly tested acute-phase proteins, cytokines, innate immune cells, and white blood cells included CRP, IL-6, IL-10, TNF- $\alpha$ and IFN- $\gamma$, T lymphocytes, various complement components (classical and alternative pathways), and natural killer (NK) cells. Some of these biomarkers, such as CRP, IL-6, TNF- $\alpha$, and IFN- $\gamma$, are known to display pro-inflammatory effects, while IL-10 is recognized as antiinflammatory in nature..$^{29,33}$ None of the reviewed studies found a statistically significant inter-group difference in IL-10.

Considering the studies that measured CRP, IL-6, TNF- $\alpha$, and IFN- $\gamma$ in PTSD sufferers versus non-PTSD sufferers, all but one found significantly higher levels of pro-inflammatory markers in the PTSD group compared with the non-PTSD group. While the outlying studies by Gola et $\mathrm{al}^{25}$ and von Känel et al $^{18}$ argued for an overall pro-inflammatory state in individuals with PTSD, they did not find (contrary to their hypothesis) significant between-group differences in proinflammatory biomarkers. However, with regard to IL-4 and IL-6, none of the included studies took into account their dual role as both pro- and anti-inflammatory cytokines. This makes interpretation of the between-group measurements of IL-4 and IL-6 levels difficult because it is unclear whether they are acting as a pro- or anti-inflammatory cytokines in PTSD. ${ }^{37,38}$

The findings highlighted in this review are clinically important in the treatment of PTSD, because if an ongoing systemic low-grade inflammatory state is consistently detected in this population, treatment may then be directed towards managing the under-regulated inflammatory aspects of the disease to provide symptom relief and potential comorbid chronic disease prevention.

\section{PTSD and chronic disease risk}

As evident in the included studies, PTSD is associated with overall elevated levels of various immune/inflammatory markers. Similarly, this pro-inflammatory state is also present in the pathogenesis of chronic physical illnesses such as CVD, insulin resistance, metabolic syndrome, pulmonary disorders, and autoimmune diseases. ${ }^{9,32}$ In the nine studies included for this review, it was the overarching view that these inflammatory processes found in both PTSD and chronic disease are related, and the catalyst for this elevated systemic inflammation may be stress induced. ${ }^{9}$

The key findings presented were that acquiring PTSD leads to an increased risk for developing physical co-morbidities, with cardiovascular, metabolic, musculoskeletal, dermatological, and pulmonary diseases being diagnosed in this population most frequently. ${ }^{39}$

From the evidence provided linking PTSD, inflammation, and chronic disease risk, it is essential to ensure that 
treatments are designed for this population such that the outcome provides a better quality of life for individuals with PTSD.

\section{Heterogeneity of included studies}

Of the studies included, it is clear that there is a link between PTSD, chronic disease risk, and a chronically pro-inflamed state. However, the large degree of heterogeneity between the studies cannot be ignored. There was a glaring absence of an included definition of "low-grade inflammation" in the majority of studies. ${ }^{18-23}$ The exclusion of this definition makes it difficult to determine the exact significance of the intergroup results in the context of inflammatory marker levels. In addition, all of the included studies measured inflammatory markers through the collection of plasma samples; however, recent research has shown that inflammatory markers collected via saliva more accurately depict the dysregulated immune state of PTSD participants. ${ }^{40}$ There was also a lack of uniformity in psychological assessment and biomarker collection methods, as well as variations in the time of day at which samples were collected. These disparities make it difficult to directly compare the studies, as different methods for assessment and collection would have differing reliability and/or sensitivity, and diurnal variance in cytokine collection would certainly confound results as cytokine concentrations fluctuate throughout the day. ${ }^{18}$

There were also inconsistencies between the studies included in this review and the confounding variables known to influence inflammation. These confounders include age, gender, ethnicity, education, marital status, employment, medications, psychiatric co-morbidities, asthma/allergies, physical co-morbidities, early life trauma, time since trauma, trauma type, BMI, alcohol/substance abuse, smoking status, and physical activity. This review recognizes that the included studies did attempt to control for various combinations of the aforementioned confounders (statistically through their results or via their inclusion/exclusion criteria) as well as the difficulty in controlling for all of these confounding factors; however, the reliability of the results still remains questionable. Without total control over the confounding variables, determining the exact cause of the demonstrated pro-inflammatory state in individuals with PTSD becomes difficult. Nonetheless, the results still displayed inconsistencies, with some studies indicating non-significance of results after controlling for certain confounding factors and the remaining studies demonstrating significance even after accounting for many of the above confounders. ${ }^{6,18-25}$ Notably, the studies that did not consistently demonstrate statistically significant findings for elevation in all inflammatory markers in the PTSD participants still reported the presence of an overall pro-inflammatory state in this population compared to their healthy counterparts. The researchers from both studies attribute this conclusion to a suggested correlation between PTSD symptom severity and TNF- $\alpha$ levels in the PTSD sample, such that the more severe the PTSD symptoms, the higher the levels of TNF- $\alpha .^{18,25}$

Based upon the limitations discussed above, it appears that there is a definite need for future studies to establish inter-study homogeneity by defining "low-grade inflammation," controlling assessment and collection methods, standardizing the time of day at which samples are collected, and accounting for the various confounding factors to the best of their abilities.

\section{Clinical applications}

There is a growing body of literature suggesting that there is a strong association between PTSD and chronic disease. Furthermore, this association may be independent of multiple confounding variables such as age, depression, and/ or co-morbid anxiety disorders. ${ }^{41}$ There is agreement that PTSD appears to involve shifts within the central nervous system and immune/inflammatory processes, such that the effects of PTSD-related chronic stress causes a dysregulation of these systems, which then incurs an elevated risk for the subsequent development of multiple chronic illnesses with an inflammatory pathophysiology. ${ }^{42,43}$

Current standard PTSD management lies within the psychiatric parameters of this disorder. ${ }^{43}$ However, there is a staggering lack of treatment targeting the co-morbid physical disease risk factors that ultimately result in overall impairment of long-term health and well-being. ${ }^{42}$ In addition, there is limited research examining acute physiological recovery from stress in PTSD sufferers. ${ }^{43}$ Such research will provide clarity on the appropriateness of their acute stress response that will help in the development of more effective treatment plans for this population.

Given that PTSD is not solely a psychiatric disorder, and the nature of its associated physical co-morbidities with inflammatory underpinnings, it is essential to develop appropriate cardiometabolic preventive therapy and treatment plans (in conjunction with therapeutic management) that reduce these long-term deleterious health outcomes.

\section{Future research}

Despite the research-based evidence of a chronic, low-grade pro-inflammatory state in individuals with PTSD and its link 
to long-term adverse health outcomes, the development of appropriate treatment methods to target these findings is yet to be established. As previously stated, the underlying link between PTSD and chronic somatic disease is illustrated by impaired psycho-physiological mechanisms leading to ongoing inflammation in these individuals. Therefore, treatment of both the psychological symptoms and physical co-morbidities in this population should address this chronically inflamed state.

\section{Conclusion}

PTSD is a severely crippling disorder that manifests in activeduty combat soldiers, military veterans, first responders and civilians alike, resulting in a number of long-term psychological and physical adverse health outcomes. The common feature underlying these conditions is a chronically elevated inflammatory state. Considering the devastating effects that PTSD has on the individual, his or her family, and society at large, there is a critical need to better understand the exact causes of PTSD and the regulatory systems/biological factors involved that contribute to the inflammatory state seen in PTSD. Once this has been achieved, implementation of an effective, easily accessible adjuvant treatment to usual care can be put in place to improve the quality of life of the PTSD population.

\section{Disclosure}

The authors report no conflicts of interest in this work.

\section{References}

1. American Psychiatric Association. Diagnostic and Statistical Manual of Mental Disorders, Fifth Edition: DSM-5. Washington DC: American Psychiatric Publishing; 2013.

2. Dennis PA, Weinberg BJ, Calhoun PS, et al. An investigation of vagoregulatory and health-behavior accounts for increased inflammation in posttraumatic stress disorder. J Psychsom Res. 2016;83:33-39.

3. Yehuda R. Post-traumatic stress disorder. $N$ Engl J Med. 2002;346:108-114.

4. Hoge CW, Castro CA, Messer SC, McGurk D, Cotting DI, Koffman RL. Combat duty in Iraq and Afghanistan, mental health problems, and barriers to care. N Engl J Med. 2004;351:13-22.

5. Foreign Affairs Defence and Trade References Committee. Mental Health of Australian Defence Force Members and Veterans. Canberra: Department of the Senate; 2016.

6. Lindqvist D, Dhabhar FS, Mellon SH, et al. Increased pro-inflammatory milieu in combat related PTSD - a new cohort replication study. Brain Behav Immun. 2017;59:260-264.

7. Babson KA, Heinz AJ, Ramirez G, et al. The interactive role of exercise and sleep on veteran recovery from symptoms of PTSD. Ment Health Phys Act. 2015;8:15-20.

8. Wolff E, Gaudlitz K, von Lindenberger BL, Plag J, Heinz A, Ströhle A. Exercise and physical activity in mental disorders. Eur Arch Psychiatry Clin Neurosci. 2011;261(Suppl 2):S186-S191.

9. Black PH. The inflammatory consequences of psychologic stress: relationship to insulin resistance, obesity, atherosclerosis and diabetes mellitus, type II. Med Hypotheses. 2006;67(4):879-891.
10. Dedert EA, Calhoun PS, Watkins LL, Sherwood A, Beckham JC. Posttraumatic stress disorder, cardiovascular, and metabolic disease: a review of the evidence. Ann Behav Med. 2010;39(1):61-78.

11. Reeves JW, Fisher AJ, Newman MG, Granger DA. Sympathetic and hypothalamic-pituitary-adrenal asymmetry in generalized anxiety disorder. Psychophysiology. 2016;53(6):951-957.

12. Daskalakis NP, Lehrner A, Yehuda R. Endocrine aspects of posttraumatic stress disorder and implications for diagnosis and treatment. Endocrinol Metab Clin North Am. 2013;42:503-513.

13. Dantzer R, O'Connor JC, Freund GG, Johnson RW, Kelley KW. From inflammation to sickness and depression: when the immune system subjugates the brain. Nat Rev Neurosci. 2008;9(1):46-56.

14. Maes M, Berk M, Goehler L, et al. Depression and sickness behavior are Janus-faced responses to shared inflammatory pathways. BMC Med. 2012;10:66.

15. Krabbe KS, Reichenberg A, Yirmiya R, Smed A, Pedersen BK, Bruunsgaard H. Low-dose endotoxemia and human neuropsychological functions. Brain Behav Immun. 2005;19(5):453-460.

16. Neigh GN, Ali FF. Co-morbidity of PTSD and immune system dysfunction: opportunities for treatment. Curr Opin Pharmacol. 2016;29:104-110.

17. Walker A, McKune A, Ferguson S, Pyne DB, Rattray B. Chronic occupational exposures can influence the rate of PTSD and depressive disorders in first responders and military personnel. Extrem Physiol Med. 2016;5:8.

18. von Känel R, Hepp U, Kraemer B, et al. Evidence for low-grade systemic proinflammatory activity in patients with posttraumatic stress disorder. J Psychiatr Res. 2007;41(9):744-752.

19. Bersani FS, Wolkowitz OM, Milush JM, et al. A population of atypical CD56- ${ }^{-}$D $16^{+}$natural killer cells is expanded in PTSD and is associated with symptom severity. Brain Behav Immun. 2016;56:264-270.

20. Heath NM, Chesney SA, Gerhart JI, et al. Interpersonal violence, PTSD, and inflammation: potential psychogenic pathways to higher $\mathrm{C}$-reactive protein levels. Cytokine. 2013;63(2):172-178.

21. Hoge EA, Brandstetter K, Moshier S, Pollack MH, Wong KK, Simon NM. Broad spectrum of cytokine abnormalities in panic disorder and posttraumatic stress disorder. Depress Anxiety. 2009;26(5):447-455.

22. Hovhannisyan LP, Mkrtchyan GM, Sukiasian SH, Boyajyan AS. Alterations in the complement cascade in post-traumatic stress disorder. Allergy Asthma Clin Immunol. 2010;6(1):3.

23. Lindqvist D, Wolkowitz OM, Mellon S, et al. Proinflammatory milieu in combat-related PTSD is independent of depression and early life stress. Brain Behav Immun. 2014;42:81-88.

24. Spitzer C, Barnow S, Völzke H, et al. Association of posttraumatic stress disorder with low-grade elevation of C-reactive protein: evidence from the general population. J Psychiatr Res. 2010;44(1):15-21.

25. Gola H, Engler H, Sommershof A, et al. Posttraumatic stress disorder is associated with an enhanced spontaneous production of pro-inflammatory cytokines by peripheral blood mononuclear cells. BMC Psychiatry. 2013;13(1):40.

26. Liberati A, Altman DG, Tetzlaff J, et al. The PRISMA statement for reporting systematic reviews and meta-analyses of studies that evaluate health care interventions: explanation and elaboration. PLoS Med. 2009;6(7):e1000100.

27. Moher D, Liberati A, Tetzlaff J, Altman D, The PRISMA Group. Preferred Reporting Items for Systematic Reviews and Meta-Analyses: The PRISMA Statement. PLoS Med. 2009;6(7):e1000097.

28. Macedo LG, Elkins MR, Maher CG, Moseley AM, Herbert RD, Sherrington $\mathrm{C}$. There was evidence of convergent and construct validity of Physiotherapy Evidence Database quality scale for physiotherapy trials. J Clin Epidemiol. 2010;63(8):920-925.

29. Verhagen AP, de Vet HC, de Bie RA, et al. The Delphi list: a criteria list for quality assessment of randomized clinical trials for conducting systematic reviews developed by Delphi consensus. J Clin Epidemiol. 1998;51:1235-1241.

30. Maher CG, Sherrington C, Herbert RD, Moseley AM, Elkins M. Reliability of the PEDro scale for rating quality of randomized controlled trials. Phys Ther. 2003;83(8):713-721. 
31. Segerstrom SC, Out D, Granger DA, Smith TW. Biological and physiological measures in health psychology. In: Benyamini Y, Johnston M, Karademas EC, editors. Assessment in Health Psychology. Boston, MA: Hogrefe; 2016:227-238.

32. Gill JM, Saligan L, Woods S, Page G. PTSD is assoicated with an excess of inflammatory immune activities. Perspect Psychiatr $C$. 2009;45(4):262-277.

33. McLeay SC, Harvey WM, Romaniuk MN, et al. Physical comorbidities of post-traumatic stress disorder in Australian Vietnam War veterans. Med J Aust. 2017;206(6):251-257.

34. Correll CU, Solmi M, Veronese N, et al. Prevalence, incidence and mortality from cardiovascular disease in patients with pooled and specific severe mental illness: a large-scale meta-analysis of 3,211,768 patients and 113,383,368 controls. World Psychiatry. 2017;16(2):163-180.

35. Groer MW, Kane B, Williams SN, Duffy A. Relationship of PTSD symptoms with combat exposure, stress, and inflammation in American soldiers. Biol Res Nurs. 2015;17(3):303-310.

36. Boscarino JA. A prospective study of PTSD and early-age heart disease mortality among Vietnam veterans: implications for surveillance and prevention. Psychosom Med. 2008;70(6):668-676.
37. Cavaillon J-M. Pro- versus anti-inflammatory cytokines: myth or reality Cell Mol Biol (Noisy-le-grand). 2001;47(4):695-702.

38. Luzina IG, Keegan AD, Heller NM, Rook GA, Shea-Donohue T, Atamas SP. Regulation of inflammation by interleukin-4: a review of "alternatives". J Leukoc Biol. 2012;92(4):753-764.

39. Britvić D, Antičević V, Kaliterna M, et al. Comorbidities with Posttraumatic Stress Disorder (PTSD) among combat veterans: 15 years postwar analysis. Int J Clin Health Psychol. 2015;15(2):81-92.

40. Wang Z, Mandel H, Levingston CA, Young MRI. An exploratory approach demonstrating immune skewing and a loss of coordination among cytokines in plasma and saliva of Veterans with combat-related PTSD. Hum Immunol. 2016;77(8):652-657.

41. Kibler JL. Posttraumatic stress and cardiovascular disease risk. JTrauma Dissociation. 2009;10(2):135-150.

42. Pace TW, Heim CM. A short review on the psychoneuroimmunology of posttraumatic stress disorder: from risk factors to medical comorbidities. Brain Behav Immun. 2011;25(1):6-13.

43. Levine AB, Levine LM, Levine TB. Posttraumatic stress disorder and cardiometabolic disease. Cardiology. 2014;127(1):1-19.
Journal of Inflammation Research

\section{Publish your work in this journal}

The Journal of Inflammation Research is an international, peer-reviewed open access journal that welcomes laboratory and clinical findings on the molecular basis, cell biology and pharmacology of inflammation including original research, reviews, symposium reports, hypothesis formation and commentaries on: acute/chronic inflammation; mediators of

\section{Dovepress}

inflammation; cellular processes; molecular mechanisms; pharmacology and novel anti-inflammatory drugs; clinical conditions involving inflammation. The manuscript management system is completely online and includes a very quick and fair peer-review system. Visit http://www.dove press.com/testimonials.php to read real quotes from published authors. 